\title{
On discernibility in symmetric languages: the case of quantum particles
}

\author{
Tomasz Bigaj ${ }^{1}$
}

Received: 5 September 2019 / Accepted: 13 February 2020 / Published online: 29 February 2020

(c) The Author(s) 2020

\begin{abstract}
In this paper I consider the question of whether absolute discernibility is attainable in symmetric languages. Simon Saunders has proven that all facts expressible in first-order language with identity can be equivalently stated within its symmetric sublanguage. I use this result to show specifically how particles of the same type can be absolutely discerned in the permutation-invariant language of the quantum theory of many particles.
\end{abstract}

Keywords Quantum particles · Absolute discernibility · Permutation invariance . Projection operators · Quantum logic

The main problem considered in this paper comes down to this simple question: is it possible to discern (in an appropriate sense of the word) objects in a language that is fully symmetric (meaning that the denotations of its formulae are permutationinvariant)? The motivation behind asking such a question derives from physics: as is well known, the quantum theory of many particles imposes a restriction on the available states of particles of the same type in the form of the requirement of their permutation invariance. As some claim, the permutation invariance of the states of composite systems leads directly to the consequence that the components of such systems can never be qualitatively discerned by their properties (in gross violation of the Leibnizian Principle of the Identity of Indiscernibles). ${ }^{1}$ In what follows I will critically evaluate this claim, citing some general facts provable within first-order logic and applying them to the specific case of quantum particles. The conclusion I am aiming to argue for is that the symmetry of a language in which we describe quantum objects of the same type does not block the possibility of making absolute discernments between them.

\footnotetext{
${ }^{1}$ The list of works in which this thesis is accepted contains, among others, French and Redhead (1988), Redhead and Teller (1992), Butterfield (1993), Huggett (2003), and French and Rickles (2003).

Tomasz Bigaj

t.f.bigaj@uw.edu.pl

1 Institute of Philosophy, University of Warsaw, ul. Krakowskie Przedmiescie 3, 00-047 Warsaw, Poland
} 
The plan of the paper is as follows. In Sect. 1 I will present and briefly discuss three fundamental notions of discernibility (absolute, relative and weak) commonly used in the literature on the subject, together with the logical relations holding among them. Section 2 contains an extended analysis of the requirement of symmetry (permutationinvariance) as applied to languages. After recalling the standard logical result showing that the symmetry of a language implies the non-existence of absolutely and relatively discerning formulas, I lay out a theorem due to Simon Saunders which implies that symmetric first-order languages are capable of expressing facts about absolute (and relative) discernibility. I also explain why the two above-mentioned results, while seemingly contradictory, are in no conflict with one another. Sections 3 and 4 are devoted to the task of applying Saunders' theorem to the case of the quantum theory of many particles. In Sect. 3 I take up the task of translating the basic elements of the quantum-mechanical formalism into a first-order language, which to my knowledge has never been done in a systematic way. I propose to introduce multi-variable predicates whose satisfaction by sequences of objects is explicated in terms of the state of the system being an eigenstate for a particular projection operator. In Sect. 4 I show directly how to use Saunders' theorem in order to argue that the symmetric language describing states of groups of 'indistinguishable' fermions and bosons contains expressions which can be interpreted as stating that particles are absolutely discernible by appropriate properties. I point out that this result hinges on the assumption that the logical connectives are to be interpreted non-classically (quantum-logically), and I briefly discuss the consequences of this assumption.

\section{Discernibility in a language}

In the first step we should define what we mean by discernibility with respect to a given language. We will present here the standard approach to this problem, expressed in the most concise way in the overview (Ladyman et al. 2012). ${ }^{2}$ Let $\mathscr{L}$ be a first-order language without proper names (constants) and without the identity symbol, whose intended semantic interpretation is given in the form of a particular relational structure $\mathcal{A}$ (consisting of a non-empty set and a number of relations of various numbers of arguments corresponding to the primitive predicates of $\mathscr{L}$ ). We will distinguish three basic types of discernibility that can be expressed in $\mathscr{L}$ under the semantic interpretation encompassed in the model $\mathcal{A}$. These types are known as absolute discernibility, relative discernibility and weak discernibility. Objects $a$ and $b$ in the domain of the model $\mathcal{A}$ are said to be absolutely discernible in $\mathscr{L}$ iff there is a one-argument formula $\Phi(x)$ in $\mathscr{L}$ such that $\Phi(x)$ is satisfied by $a$ and not by $b$ in $\mathcal{A} \cdot{ }^{3}$ Objects $a$ and $b$ are relatively discernible in $\mathscr{L}$ iff there is a two-argument formula $\Phi(x, y)$ in $\mathscr{L}$ such that $\Phi(x, y)$ is satisfied in $\mathcal{A}$ by pair $(a, b)$ and not by $(b, a)$. And, thirdly, objects $a$ and $b$ are weakly discernible in $\mathscr{L}$ iff there is a formula $\Phi(x, y)$ in $\mathscr{L}$ such that $\Phi(x, y)$ is

\footnotetext{
2 Many important results reported in Ladyman et al. (2012) were proven in Ketland (2006, 2011). See also Bigaj (2015a) for some follow-up discussions and extensions of Ladyman et al. (2012).

3 Beware: symbols " $a$ " and " $b$ " do not belong to language $\mathscr{L}$, but to the metalanguage in which we describe semantical relations between $\mathscr{L}$ and the corresponding relational structure $\mathcal{A}$. Thus we are not violating the assumption that $\mathscr{L}$ does not contain any proper names.
} 
satisfied in $\mathcal{A}$ by pairs $(a, b)$ and $(b, a)$, and is not satisfied by pairs $(a, a)$ and $(b, b){ }^{4}$ These three definitions can be concisely given as follows ${ }^{5}$ :

Definition 1.1 Let $a$ and $b$ be elements of the domain of structure $\mathcal{A}$. Then

(a) $a$ and $b$ are absolutely discernible in $\mathscr{L}$ iff there is an open formula $\Phi(x)$ in $\mathscr{L}$ such that $\mathcal{A} \vDash \Phi(a)$ and $\mathcal{A} \vDash \neg \Phi(b),{ }^{6}$

(b) $a$ and $b$ are relatively discernible in $\mathscr{L}$ iff there is an open formula $\Phi(x, y)$ in $\mathscr{L}$ such that $\mathcal{A} \vDash \Phi(a, b)$ and $\mathcal{A} \vDash \neg \Phi(b, a)$,

(c) $a$ and $b$ are weakly discernible in $\mathscr{L}$ iff there is an open formula $\Phi(x, y)$ in $\mathscr{L}$ such that $\mathcal{A} \vDash \Phi(a, b)$ and $\mathcal{A} \vDash \neg \Phi(a, a)$.

We can also define what it means for a particular formula in $\mathscr{L}$ to discern two objects-absolutely, relatively or weakly.

Definition 1.2 Let $a$ and $b$ be elements of the domain of structure $\mathcal{A}$. Then

(a) formula $\Phi(x)$ in $\mathscr{L}$ absolutely discerns objects $a$ and $b$ iff $\mathcal{A} \vDash \Phi(a)$ and $\mathcal{A} \vDash$ $\neg \Phi(b)$, or $\mathcal{A} \vDash \Phi(b)$ and $\mathcal{A} \vDash \neg \Phi(a)$,

(b) formula $\Phi(x, y)$ in $\mathscr{L}$ relatively discerns objects $a$ and $b$ iff $\mathcal{A} \vDash \Phi(a, b)$ and $\mathcal{A}$ $\vDash \neg \Phi(b, a)$, or $\mathcal{A} \vDash \Phi(b, a)$ and $\mathcal{A} \vDash \neg \Phi(a, b)$,

(c) formula $\Phi(x, y)$ in $\mathscr{L}$ weakly discerns objects $a$ and $b$ iff $\mathcal{A} \vDash \Phi(a, b), \mathcal{A} \vDash \Phi(b$, $a), \mathcal{A} \vDash \neg \Phi(a, a)$ and $\mathcal{A} \vDash \neg \Phi(b, b)$.

The second disjunct in (a) is necessary in order to make sure that discernibility by a formula is a symmetric relation: if $\Phi$ discerns $a$ from $b$, it would be rather absurd

\footnotetext{
4 The definition of weak discernibility can be simplified by eliminating the parts requiring the satisfaction of formula $\Phi$ by the pair $(b, a)$ and the non-satisfaction of $\Phi$ by $(b, b)$. If $\Phi$ is known to be satisfied by ( $a$, $b$ ) and not satisfied by $(a, a)$, we can define a new weakly discerning formula $\Psi(x, y)$ whose form depends on whether $(b, b)$ actually satisfies $\Phi$ or not. If $\Phi$ is not satisfied by $(b, b)$, the formula $\Psi(x, y):=\Phi(x, y)$ $\vee \Phi(y, x)$ will be weakly discerning in the above-defined sense (i.e. it will be symmetric and irreflexive in the set $\{a, b\})$. And if $\Phi$ is satisfied by $(b, b)$, it can be checked that the following formula will do the job of weakly discerning $a$ and $b:[\Phi(x, y) \vee \Phi(y, x)] \wedge[\neg \Phi(x, x) \vee \neg \Phi(y, y)]$.

5 The expressions of the form $\mathcal{A} \vDash \Phi(a)$ and $\mathcal{A} \vDash \Phi(a, b)$ used in Definition 1.1 are abbreviations of the more standard symbolization of the relation of satisfaction: $\mathcal{A} \vDash \Phi(x)[a]$ and $\mathcal{A} \vDash \Phi(x, y)[a, b]$. The latter makes it clear that symbols " $a$ " and " $b$ " are not parts of the formula $\Phi$, since they do not belong to language $\mathscr{L}$ but to the metalanguage.

6 Sometimes two further subcategories of absolute discernibility are distinguished: absolute intrinsic discernibility and absolute extrinsic discernibility. Intrinsic discernibility restricts formulas $\Phi$ to those that do not contain quantifiers, while extrinsic discernibility admits quantifiers in discerning formulas. The standard gloss on these concepts is that intrinsic discernibility is in terms of intrinsic properties only (properties not involving other objects), whereas extrinsic discernibility admits relations with other entities as a means to discern two objects. The reason why extrinsic absolute discernibility is defined in terms of quantifiers is that in a language with no constants the only way to 'contract' a multi-variable formula (for instance $\Phi(x, y)$ ) to a one-argument formula is by binding all variables except one by quantifiers, as in $\exists y \Phi(x, y)$, or by replacing different variables by the same symbol, as in $\Phi(x, x)$. (However, the second method produces a formula which, even though technically represents a relation of an object to itself, is not extrinsic in the sense of involving other objects.) Thus, if a one-argument formula with no constants does not contain quantifiers, it cannot involve reference to other objects and therefore does not represent an extrinsic property. In the context of quantum-mechanical indiscernibility both concepts are important, as the typical arguments for the indiscernibility of 'identical' quantum particles employ both non-relational and relational properties [see e.g. French and Redhead (1988), Butterfield (1993), and Dieks and Versteegh (2008).
} 
to deny that $\Phi$ discerns $b$ from $a$. Also, we want to ensure that if $\Phi$ discerns two objects, so does its negation $\neg \Phi$. Similarly, the second clause of the disjunction in (b) ensures that discernibility by a formula is symmetric (even though in this case the discerning relation is clearly asymmetric-beware of conflating the cognate notions of being relatively discerned by a formula and being connected by a relation that does the discerning). In case (c) it is unnecessary to add the second clause, since the relation of weak discernibility is already stipulated to be symmetric.

Instead of considering the purely 'qualitative' language $\mathscr{L}$, we could expand it to $\mathscr{L}^{*}$ by adding either a proper name for every element in the domain (given the assumption that the intended model $\mathcal{A}$ is finite), or by adding the symbol of identity $=$ with its intended interpretation $\left\{\left(a_{1}, a_{1}\right),\left(a_{2}, a_{2}\right), \ldots\left(a_{n}, a_{n}\right)\right\}$ to $\mathscr{L}^{=}$. That way we will obtain new languages $\mathscr{L}^{*}$ and $\mathscr{L}^{=}$and new corresponding notions of discernibility. All in all, we have introduced nine distinct grades of discernibility, which turn out to be mutually connected by relations of logical entailment. These relations can be synthetically presented in the following theorem (the abbreviations used in the theorem should be self-explanatory $)^{7}$ :

Theorem 1.1 The following logical relations hold:

(a) $\operatorname{Abs}_{\mathscr{L}} \Rightarrow \operatorname{Rel}_{\mathscr{L}} \Rightarrow \operatorname{Weak}_{\mathscr{L}}$

(b) $\operatorname{Abs} \mathscr{L} \Rightarrow \operatorname{Abs} \mathscr{L}=$

(c) $\operatorname{Rel}_{\mathscr{L}} \Rightarrow \operatorname{Rel}_{\mathscr{L}}=$

(d) $\operatorname{Abs} \mathscr{L}=\Rightarrow \operatorname{Rel}_{\mathscr{L}} \Rightarrow$ Weak $\mathscr{L}$

(e) $\operatorname{Weak}_{\mathscr{L}} \Leftrightarrow \operatorname{Weak}_{\mathscr{L}} * \operatorname{Abs}_{\mathscr{L}} * \Leftrightarrow \operatorname{Rel}_{\mathscr{L}} *$

(f) Weak $\mathscr{L}=\Leftrightarrow \neq$

(g) No implications other than those entailed by (a)-(f) hold true.

Of particular importance to us are clauses (a) and (d) which show (together with (e) and (g)) that weak discernibility is indeed the weakest of all typical grades of discernibility, with absolute discernibility in $\mathscr{L}$ the strongest of all. Clause (e) shows that in a language equipped with proper names for every element of the domain all grades of discernibility collapse into weak discernibility. Clause (f) establishes that weak discernibility in a language with identity is trivially non-identity.

\section{Discernibility and symmetry}

It is common knowledge that the language in which we are supposed to describe systems of quantum particles of the same type should satisfy the requirement of symmetry, also known as permutation invariance. Of course, the primary reason for the symmetry of a language is that the corresponding reality described by the language is supposed to be symmetric too. Thus, in the case of the language of quantum mechanics its symmetry follows from the fact that the physical states of particles of the same type described in this language are supposed to be permutation-invariant. Due to the correspondence between language $\mathscr{L}$ and its intended interpretation $\mathcal{A}$, we can move

7 For proofs of these facts see Ladyman et al. (2012) and references therein. 
back and forth between the symmetry of the language and the symmetry of its corresponding model. But what does it precisely mean for a language to be permutation invariant (symmetric)? The standard definition can be given as follows.

Definition 2.1 Let $\mathscr{L}$ be a first-order language and $\mathcal{A}$ its intended interpretation. Let $\sigma: \operatorname{Dom}(\mathcal{A}) \rightarrow \operatorname{Dom}(\mathcal{A})$ be a permutation of the domain of $\mathcal{A}$ (that is, a bijection of $\operatorname{Dom}(\mathcal{A})$ onto itself). Then $\mathscr{L}$ is symmetric iff for every open formula $\Phi\left(x_{1}, x_{2}, \ldots x_{n}\right)$ in $\mathscr{L}$ and any permutation $\sigma, \mathcal{A} \vDash \Phi\left(a_{1}, a_{2}, \ldots a_{n}\right)$ iff $\mathcal{A} \vDash \Phi\left(\sigma\left(a_{1}\right), \sigma\left(a_{2}\right), \ldots \sigma\left(a_{n}\right)\right){ }^{8}$

It can be proven relatively easily (by induction over the complexity of formulas) that Definition 2.1 is equivalent to the condition of symmetry imposed on the structure $\mathcal{A}$ corresponding to $\mathscr{L}$, spelled out as follows:

Definition 2.2 Relational structure $\mathcal{A}$ is symmetric iff for any $k$-element relation $R$ in $\mathcal{A}$, any elements $a_{1}, \ldots, a_{k} \in \operatorname{Dom}(\mathcal{A})$ and any permutation $\sigma: \operatorname{Dom}(\mathcal{A}) \rightarrow \operatorname{Dom}(\mathcal{A})$, $R a_{1} \ldots a_{k}$ iff $R \sigma\left(a_{1}\right) \ldots \sigma\left(a_{k}\right)$.

The following fact regarding discernibility in symmetric languages holds:

Theorem 2.1 Let $\mathscr{L}$ be a symmetric language (in the sense of Definition 2.1). Then no two objects in $\operatorname{Dom}(\mathcal{A})$ are absolutely or relatively discerned in $\mathscr{L}$.

The proof of this theorem is quick. Suppose that elements $a$ and $b$ of the domain $\operatorname{Dom}(\mathcal{A})$ are relatively discerned in $\mathscr{L}$. That means, according to Definition 1.1. (b) that there is a formula $\Phi(x, y)$ in $\mathscr{L}$ such that $\mathcal{A} \vDash \Phi(a, b)$ and $\mathcal{A} \vDash \neg \Phi(b, a)$, but this directly contradicts Definition 2.1, since the transposition $\sigma(a)=b$ and $\sigma(b)$ $=a$ does not preserve the satisfaction of formula $\Phi(x, y)$. Thus $a$ and $b$ can't be relatively discerned in $\mathscr{L}$. And because of Theorem 1.1 (a), this implies that $a$ and $b$ are not absolutely discernible either. Theorem 2.1 remains also valid for languages with identity $\mathscr{L}^{=}$when we limit ourselves to finite models, but its proof is more complicated (see Caulton and Butterfield 2012, Sect. 4.3).

On the other hand, the symmetry of $\mathscr{L}$ does not exclude the possibility of weak discernment, as seen in the following example. In the two-element symmetric graph given in Fig. 1, the double arrow represents a relation corresponding to a primitive, two-place predicate $P$ in $\mathscr{L}$ (note that the relation used in the graph is irreflexive, as no object is connected to itself by an arrow). Clearly, the graph is invariant under the permutation of objects, and yet they are weakly discernible by formula $P(x, y) .{ }^{9}$

One lesson from this basic logical analysis seems to be inescapable: if we have at our disposal a symmetric language only, we can at best achieve weak discernibility

\footnotetext{
${ }^{8}$ It is conceivable to introduce an alternative notion of symmetry (permutation-invariance) with respect to languages, which would be based not on permutations of objects in the domain, but on permutations of their occurrences within a given formula. A definition of such a notion would look similar to Definition 2.1 , except that now $\sigma$ would signify a permutation of the set of numbers $\{1, \ldots, n\}$, and the condition of permutation-invariance would be given in the form of the following equivalence: $\mathcal{A} \vDash \Phi\left(a_{1}, a_{2}, \ldots a_{n}\right)$ iff $\mathcal{A} \vDash \Phi\left(a_{\sigma(1)}, a_{\sigma(2)}, \ldots a_{\sigma(n)}\right)$. The difference between the two concepts of symmetry can be illustrated with the help of a simple example. Suppose that a three-argument formula $\Phi(x, y, z)$ is satisfied by the triple $(a, a, b)$ in a two-element domain $\{a, b\}$. According to Definition 2.1, the condition of symmetry demands only that $\mathcal{A} \vDash \Phi(b, b, a)$, whereas the new definition instead implies that $\mathcal{A} \vDash \Phi(b, a, a)$ and $\mathcal{A} \vDash \Phi(a, b, a)$. However, it turns out that the alternative definition of symmetry is rather uninteresting, since its satisfaction
} 


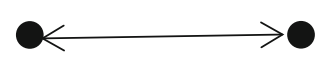

Fig. 1 A two-element symmetric graph

but not relative and not absolute discernibility. This conclusion apparently supports the orthodox approach to the question of the discernibility of quantum particles of the same type. It is commonly accepted that in the permutation-invariant language of the quantum theory of many particles no absolute discernibility is possible, but weak discernibility is sometimes attainable. ${ }^{10}$ However, in what follows we will try to cast doubt on the inevitability of this conclusion. We will start with a relatively little-known theorem due to Saunders (2006b, 2013), which, on the face of it, seems to contravene the conclusion that symmetry prevents absolute discernment.

Theorem 2.2 Let $\mathscr{L}^{=}$be a first-order language without proper names but with the identity symbol. Then for every sentence $T$ in $\mathscr{L}^{=}$and every natural number $N$, there is a sentence $S$ in $\mathscr{L}^{=}$of the form $S=\exists x_{1} \ldots \exists x_{n} G\left(x_{1}, \ldots, x_{n}\right)$ such that predicate $G$ is symmetric, and $S$ is equivalent to $T$ in all models of cardinality $N$.

The sketch of a proof for this theorem is as follows (for details see Saunders 2006b, pp. 209-210). Every sentence $T$ can be presented in the standard prenex form as $Q_{1}, \ldots$, $Q_{n} F\left(x_{1}, \ldots, x_{n}\right)$, where $Q_{i}$ is either $\exists x_{i}$ or $\forall x_{i}$. In order to construct the corresponding symmetric sentence $S$, we eliminate every quantifier $Q_{i}$ step by step, starting with $Q_{n}$, while simultaneously replacing formula $F\left(x_{1}, \ldots, x_{n}\right)$ with either a conjunction (when $Q_{i}$ is the universal quantifier) or a disjunction (when $Q_{i}$ is the existential quantifier) of formulas $F\left(\ldots a_{1} \ldots\right), \ldots, F\left(\ldots a_{N} \ldots\right)$, where all occurrences of the variable $x_{i}$ are replaced with unique names $a_{1}, \ldots, a_{N}$ of all elements in the domain. For instance, the first step in the procedure in the case when $Q_{n}$ is universal will give us formula

$Q_{1} \ldots Q_{n-1}\left[F\left(x_{1}, \ldots, x_{n-1}, a_{1}\right) \wedge F\left(x_{1}, \ldots, x_{n-1}, a_{2}\right) \wedge \ldots \wedge F\left(x_{1}, \ldots, x_{n-1}, a_{N}\right)\right]$.

After finishing this procedure, we end up with a formula with no quantifiers but instead containing constants $a_{1}, \ldots, a_{N}$. Finally, we replace every occurrence of $a_{i}$ with a variable $x_{i}$ bound by an existential quantifier, and we add to the entire sentence the formula stating that all $x_{i}$ 's are distinct and that they exhaust the entire domain (every object in the domain is identical with some $x_{i}$ ). The sentence $S$ obtained during this procedure is symmetric by design, and it is also not difficult to observe that it must be equivalent to $T$ in all models of cardinality $N$.

Theorem 2.2 seems to be highly relevant to the issue of the relation between symmetry and discernibility. Suppose that $\mathscr{L}^{=}$is a language in which all elements of a

\section{Footnote 8 continued}

by a language $\mathscr{L}$ implies that all predicates in $\mathscr{L}$ are either empty or full. No interesting theory can be formulated in a language whose predicates are either satisfied by all objects, or by none.

9 This example easily generalizes for an arbitrary number of objects. In a structure consisting of one binary relation $R$ such that it holds between any two distinct objects and does not hold between any object and itself (i.e. it is true that $\forall x y(x \neq y \leftrightarrow R x y))$, the relation $R$ weakly discerns every two objects in spite of the fact that the structure is clearly permutation-invariant.

10 The possibility of the weak discernment of quantum particles has been extensively studied e.g. in Saunders (2003, 2006a), Muller and Saunders (2008), Muller and Seevinck (2009), Muller (2011, 2015), Caulton (2013), Huggett and Norton (2014), Bigaj (2015b). 
(finite) domain $\operatorname{Dom}(\mathcal{A})$ are absolutely discernible. Then, Theorem 2.2 ensures that all true sentences about this domain expressible in $\mathscr{L}^{=}$can be equivalently formulated in its symmetric sublanguage $\operatorname{Sym}\left(\mathscr{L}^{=}\right)$consisting of permutation-invariant formulas only. And since the collection of all sentences of $\mathscr{L}^{=}$true in $\mathcal{A}$ presumably include truths regarding absolute discernibility of objects in $\operatorname{Dom}(\mathcal{A})$, it follows that these truths are expressible in the symmetric language $\operatorname{Sym}\left(\mathscr{L}^{=}\right)$. However, we have to be careful here. The way we defined absolute discernibility in Definition 1.1 (a) does not automatically ensure that facts regarding the absolute discernibility of objects will be expressible in language $\mathscr{L}^{=}$, since the definition is given in the metalanguage. And, as we learn from the well-known limitation theorems about the non-definability of truth or consistency, not all metalinguistic facts about a given language (or a theory formulated in that language) can be expressed in the language (theory) itself. In particular, even though objects $a$ and $b$ are absolutely discernible by formula $\Phi(x)$ in $\mathscr{L}^{=}$, this very truth may not be expressible in $\mathscr{L}$ due to the simple fact that $\mathscr{L}$ does not contain proper names for $a$ and $b$.

Approaching this problem more generally, we may ask whether there is a sentence $A b s$ in $\mathscr{L}^{=}$which states that all objects in the finite domain of $\mathscr{L}$ are absolutely discernible from each other. And it is easy to see that the answer to this question is "yes". Let the elements of the domain be symbolized as $\left\{a_{1}, \ldots, a_{N}\right\}$. By assumption, for every pair $\left(a_{i}, a_{j}\right)$ such that $i \neq j$ there is a formula $\Phi_{i j}(x)$ in $\mathscr{L}^{=}$that absolutely discerns $a_{i}$ and $a_{j}$, that is $\mathcal{A} \vDash\left[\Phi_{i j}\left(a_{i}\right) \wedge \neg \Phi_{i j}\left(a_{j}\right)\right] \vee\left[\Phi_{i j}\left(a_{j}\right) \wedge \neg \Phi_{i j}\left(a_{i}\right)\right]$. If we take the disjunction of all such formulas over all possible $i \neq j$, it is clear that the resulting formula must be satisfied by every pair of distinct objects, and that the satisfaction of the universal generalization of this formula is equivalent to the statement that all distinct objects are absolutely discernible. Hence, the sought-after sentence is as follows:

(1) $A b s \equiv \forall x \forall y\left\{x \neq y \rightarrow \bigvee_{i \neq j}^{N}\left[\Phi_{i j}(x) \wedge \neg \Phi_{i j}(y)\right] \vee\left[\Phi_{i j}(y) \wedge \neg \Phi_{i j}(x)\right]\right\} .{ }^{11}$

Now we can apply Saunders' procedure from Theorem 2.2 to the rhs of formula (1), eliminating every universal quantifier and adding an $N$-argument conjunction of formulas with constants to obtain the following:

(2) $\bigwedge_{k, l=1}^{N}\left\{a_{k} \neq a_{l} \rightarrow \bigvee_{i \neq j}^{N}\left[\Phi_{i j}\left(a_{k}\right) \wedge \neg \Phi_{i j}\left(a_{l}\right)\right] \vee\left[\Phi_{i j}\left(a_{l}\right) \wedge \neg \Phi_{i j}\left(a_{k}\right)\right]\right\}$

Finally, we will obtain the totally symmetric formula logically equivalent (in $N$ element domains) to the original statement $A b s$ :

(3) $\exists x_{1} \ldots \exists x_{N}\left\{\rho\left(x_{1}, \ldots, x_{N}\right) \wedge \bigwedge_{k \neq l}^{N}\left\{x_{k} \neq x_{l} \rightarrow \bigvee_{i \neq j}^{N}\left[\Phi_{i j}\left(x_{k}\right) \wedge \neg \Phi_{i j}\left(x_{l}\right)\right] \vee\right.\right.$ $\left.\left.\left[\Phi_{i j}\left(x_{l}\right) \wedge \neg \Phi_{i j}\left(x_{k}\right)\right]\right\}\right\}$

where $\rho\left(x_{1}, \ldots, x_{N}\right)$ abbreviates the following: $\bigwedge_{i \neq j}^{N} x_{i} \neq x_{j} \wedge \forall x \bigvee_{k=1}^{N} x=x_{k}$, that is the formula stating that there are exactly $N$ objects in the domain. ${ }^{12}$

\footnotetext{
11 It may appear, at first glance, that the disjunctive formula in (1) is already symmetric, since it remains invariant under the exchange of variables $x$ and $y$, so there is no need to appeal to Saunders' Theorem 2.2. But this is incorrect. Symmetry, as defined in Definition 2.1, implies that if a two-argument formula is satisfied by the pair $(a, b)$ of distinct objects, it must be satisfied by every other pair of distinct objects. This is by no means guaranteed by the disjunctive form of the expression in (1).

12 Technically, the antecedent condition $x_{k} \neq x_{l}$ of the implication in (3) can be dropped, due to the presence of the condition $\rho\left(x_{1}, \ldots, x_{N}\right)$.
} 
A similar result obtains in the case of relative discernibility. That is, if all objects are relatively discernible in language $\mathscr{L}^{=}$, then the following sentence expresses this very fact in finite domains of cardinality $N$ (formula $\Psi_{i j}(x, y)$ is assumed to relatively discern objects $a_{i}$ and $a_{j}$ ):

$$
R e l \equiv \forall x \forall y\left\{x \neq y \rightarrow \bigvee_{i \neq j}^{N}\left[\psi_{i j}(x, y) \vee \psi_{i j}(y, x)\right]\right\}
$$

The rhs of the equivalence (4) after symmetrization will look similar to (3):

$$
x_{1} \ldots \exists x_{N}\left\{\rho\left(x_{1}, \ldots, x_{N}\right) \wedge \bigwedge_{k \neq l}^{N}\left\{x_{k} \neq x_{l} \rightarrow \bigvee_{i \neq j}^{N}\left[\psi_{i j}\left(x_{k}, x_{l}\right) \wedge \psi_{i j}\left(x_{l}, x_{k}\right)\right]\right\}\right.
$$

Now we are in a position to explain away the apparent inconsistency between Theorems 2.1 and 2.2. The impossibility of absolute (and relative) discernibility in symmetric languages, as stated in Theorem 2.1, means that there is no symmetric formula that would discern objects absolutely or relatively. Nevertheless, this does not preclude the possibility that there may be a symmetric sentence expressing the fact that objects are absolutely (or relatively) discernible in a broader, non-symmetric language. Sentences (1) and (2) are constructed with the help of symmetric combinations of predicates from a non-symmetric language $\mathscr{L}^{=}$, but they don't assert the possibility of absolute (relative) discernibility in $\operatorname{Sym}\left(\mathscr{L}^{=}\right)$, but in $\mathscr{L}^{=}$. The possibility of conveying in a symmetric language the fact that some objects are absolutely discernible in a different, non-symmetric language, does not invalidate the fact, stated in Theorem 2.1, that absolute discernment, as defined in Definition 1.1, cannot be literally achieved in a symmetric language. However, if sentences (3) or (5) are true, we are justified in claiming that objects in the domain are in fact discernible by appropriate properties or relations, even though these properties and relations cannot find their direct linguistic representations in $\operatorname{Sym}\left(\mathscr{L}^{=}\right) .{ }^{13}$ The issue of the appropriateness of these properties and relations in the case of the quantum theory of many particles will be discussed in the next section.

\section{Quantum-mechanical predicates}

The next logical step should be to apply the general analysis done above to the specific case of systems of many particles obeying symmetry restrictions. The idea is of course to see whether absolute discernibility can be proven to be satisfied in the case of particles occupying permutation-invariant states, following the method from Theorem 2.2. However, when attempting to do this, we immediately encounter a serious stumbling block. The problem is that there is no straightforward way to cast the quantum-mechanical formalism in terms of first-order logic with its well-known structure of multi-variable predicates and logical constants. So far no rigorous and systematic method of translating the quantum-mechanical framework into standard first-order logic has been proposed, apart from some rather limited in scope and ad hoc transla-

\footnotetext{
13 If we wanted, we could call this type of discernibility "implicit". However, to avoid possible confusion, we have to stress that implicit absolute (or relative) discernibility is not a new grade of discernibility, but the old one in a new guise. The concept of "implicit" is relative to a language-what counts as regular absolute discernibility in language $\mathscr{L}^{=}$is classified as implicit absolute discernibility in $\operatorname{Sym}\left(\mathscr{L}^{=}\right)$.
} 
tions used for specific purposes. ${ }^{14}$ In what follows we will try to approach this problem more generally, but the result will be partially negative in that it will be shown that certain translations cannot be done for some rather fundamental reasons. However, what can be done should be sufficient for the purpose of answering the main question of this article.

Before we move on to the specific task of constructing a first-order language for the quantum theory of many particles (QTMP), one general remark is in order. As we have seen, Saunders' Theorem 2.2 assumes that the considered language does not contain proper names (individual constants). This may be seen as contradicting the standard practice of QTMP, which commonly uses indices (labels) as names of individual particles. In particular, the requirement of permutation invariance is typically cast in a language that uses labels as individual constants. However, as we will see below, the presence of individual constants is by no means necessary to express the requirement of permutation invariance in quantum mechanics. The permutation invariance of a particular formula can be expressed in the metalanguage, as in Definition 2.1, where only metalinguistic terms are used. Thus there is no reason to believe that Saunders' theorem is inapplicable to the case of the language of QTMP. ${ }^{15}$

We will start with the simplest case of one quantum-mechanical system with no proper parts. The standard way to formally represent a specific property ${ }^{16}$ of this system is with the help of a particular subspace of the entire Hilbert space of states for this system or, equivalently, of a projector onto that subspace (there is a one-toone correspondence between orthogonal projectors and subspaces onto which they project). That is, the fact that a given physical system possesses a particular property is formally expressed by saying that its state vector $|v\rangle$ lies in a given subspace $\mathcal{V}$ of $\mathscr{H}$ (or, alternatively, that $|v\rangle$ is an eigenstate of the corresponding projector $P_{\mathcal{V}}$ with the eigenvalue 1). ${ }^{17}$ In the case when $\mathcal{V}$ is one-dimensional (a ray) we say that the property is categorical (it amounts to the fact that there is a maximal, non-degenerate observable $O$ whose value is determined when vector $|v\rangle$ lies in $\mathcal{V})$. But $\mathcal{V}$ may be more than one-dimensional, in which case the property is less specific, given the assumption that $O$ is non-degenerate (in such a case it is usually assumed that the value of a particular observable $O$ lies within a certain range of possible values rather than being determined sharply).

Given that we are considering the domain containing one object only, we can safely limit ourselves to monadic predicates in our first-order language reconstruction. And there is a natural and simple correspondence between properties represented

\footnotetext{
14 One such attempt has been made in Muller and Seevinck (2009) in which the authors defined a relation that weakly discerns fermions of the same type. I will use some of their insights in my subsequent analysis. 15 I am grateful to an anonymous referee for pressing me on that point.

16 By a 'specific property' I understand a particular range of values of a given quantity, and not the quantity itself (e.g. spin, position, momentum). Moreover, I limit myself to so-called state-dependent properties, leaving aside state-independent ones, such as rest mass or charge, which are not representable with the help of Hermitian operators.

17 We can easily generalize this approach to cover states that are represented not by vectors (rays) but by density operators (convex sums of projectors). In that case the system possesses the property associated with $P_{\mathcal{V}}$ iff the range of the density operator representing the state of the system lies within subspace $\mathcal{V}$.
} 
by projectors and one-argument predicates satisfiable by the considered system. Let $\Phi_{P_{\mathcal{V}}}(\xi)$ be a monadic predicate in the language that we are constructing such that

(6) $\Phi_{P_{\mathcal{V}}}(\xi)$ is satisfied by the system $a$ iff $a$ is in a state $|v\rangle$ such that $P_{\mathcal{V}}|v\rangle=|v\rangle .{ }^{18}$

In other words, $\Phi_{P_{\mathcal{V}}}(a)$ is true, if the vector representing the state of system $a$ lies in subspace $\mathcal{V}$ (we are presupposing the eigenstate-eigenvalue link here). If we assume that for each projector there is a corresponding predicate, we can express in our language any simple sentence regarding the possession of quantum-mechanical properties by the system. One small problem with this assumption is that we may have to accept the existence of an infinite (in fact uncountably infinite) number of primitive predicates. Alternatively, we may introduce one two-place predicate $\Phi(\xi, \mathcal{V})$ whose truth conditions are exactly as stated in the rhs of the equivalence in (6). Finally, if one wishes, one can use a three-place predicate $\Phi(\xi, \mathcal{V}, \varphi)$ which is satisfied by an object $a$, a subspace $\mathcal{V}$ and a state $|\varphi\rangle$ iff $|\varphi\rangle$ is the state of $a$ and $|\varphi\rangle \in \mathcal{V}$.

Now we will have to extend our language to describe systems of many particles. Suppose, then, that we have $N$ particles jointly forming a system described by a state vector $|\varphi(1, \ldots, N)\rangle$. Our goal will be to define predicates with arity $k$ smaller or equal $N$. The idea, again, is that these predicates should correspond to appropriate projectors acting in the tensor product $\mathscr{H}^{N}=\mathscr{H}_{1} \otimes \ldots \otimes \mathscr{H}_{N}$. However, we have to proceed cautiously, since concrete projectors are already 'attached' to specific factor Hilbert spaces, and predicates in formalized language should be satisfiable by various combinations of objects. Thus we cannot tie a given $k$-argument predicate to a particular projector, but rather to an entire class of projectors that share their 'physical' meaning while being attributed to different objects in the composite system. This can be done as follows. Let us start with a particular subset of the set of $N$ objects which can be picked using simply numbers as labels: $1, \ldots, k$ (keep in mind that these are labels that belong to the metalanguage, not the first-order language that we are constructing). A specific property of this subset can be represented by any projector that is the product of $k$ projectors acting in the first $k$ Hilbert spaces, and $N-k$ identity operators acting in the remaining spaces: $P_{1}^{\left(a_{1}\right)} \otimes \ldots \otimes P_{k}^{\left(a_{k}\right)} \otimes I_{k+1} \otimes \ldots \otimes I_{N}$. In addition to that, some linear combinations of projectors of this type can also be permitted, even though not all such combinations will produce projectors, which are idempotent operators. ${ }^{19}$ Generally, all permissible operators representing properties of the first $k$ particles can be written in the following form:

(7) $\Omega_{k}=P(1, \ldots, k) \otimes I(k+1, \ldots, N)$,

where $P(1, \ldots, k)$ is any projector acting in the $k$-fold tensor product of Hilbert spaces $\mathscr{H}_{1} \otimes \ldots \otimes \mathscr{H}_{k}$, and $I(k+1, \ldots, N)$ is the product of $N-k$ identity operators.

The $k$-argument predicate $\Phi_{\Omega_{k}}$ corresponding to the above projector $\Omega_{k}$ has to be built step by step. In the first step we specify the truth condition for $\Phi_{\Omega_{k}}$ applied to particles $1, \ldots, k$ as follows:

\footnotetext{
18 Following a referee's suggestion, I am using the symbols $\xi_{1}, \ldots \xi_{n}$ as variables of the first-order language ranging over particles, instead of the standard notation $x_{1}, \ldots, x_{n}$, since the latter symbols are often used as representing spatial coordinates of the wave function.

19 One condition ensuring that a particular linear combination of projectors will be idempotent is that single-particle projectors participating in the combination should be mutually orthogonal.
} 
(8) $\Phi_{\Omega_{k}}\left(\xi_{1}, \ldots, \xi_{k}\right)$ is satisfied by the $k$-tuple $(1, \ldots, k)$ iff the $N$-element system is in a state $|\varphi\rangle$ such that $\Omega_{k}|\varphi\rangle=|\varphi\rangle .^{20}$

In order to stipulate the condition of the satisfaction of $\Phi_{\Omega_{k}}$ for any other $k$-tuple, we have to transform the projector $\Omega_{k}$ accordingly. That is, let $\sigma$ be any permutation of the set of metalinguistic labels $\{1, \ldots, N\}$. In that case we stipulate the following to be true:

(9) $\Phi_{\Omega_{k}}\left(\xi_{1}, \ldots, \xi_{k}\right)$ is satisfied by the $k$-tuple $(\sigma(1), \ldots, \sigma(k))$ iff the $N$-element system is in a state $|\varphi\rangle$ such that $\sigma^{-1} \Omega_{k} \sigma|\varphi\rangle=|\varphi\rangle$.

As can be seen from the above, the satisfaction of the predicate $\Phi_{\Omega_{k}}$ by any sequence of objects other than $1, \ldots, k$ requires that we transform the operator $\Omega_{k}$ to be applicable to this sequence. That way we can cover all cases of satisfaction of predicate $\Phi_{\Omega_{k}}$ by $k$-element sequences formed from the set $\{1, \ldots, N\}$ with no repetitions. However, there remains cases in which $\Phi_{\Omega_{k}}$ is applied to sequences with repetitions. And these cases present us with a difficulty that ultimately cannot be overcome in full generality, except in some special cases. Let us explain what we are up against here using the simplest possible case of two particles $(N=2)$. Let $\Omega^{(2)}$ be any projector acting in the product $\mathscr{H}_{1} \otimes \mathscr{H}_{2}$. The corresponding two-argument predicate $\Phi_{\Omega^{(2)}}\left(\xi_{1}, \xi_{2}\right)$ will receive the following partial characteristics:

(10) $\Phi_{\Omega^{(2)}}\left(\xi_{1}, \xi_{2}\right)$ is satisfied by pair $(1,2)$ iff the system is in a state $|\varphi(1,2)\rangle$ such that $\Omega^{(2)}|\varphi(1,2)\rangle=|\varphi(1,2)\rangle$;

$\Phi_{\Omega^{(2)}}\left(\xi_{1}, \xi_{2}\right)$ is satisfied by pair $(2,1)$ iff the system is in a state $|\varphi(1,2)\rangle$ such that $P_{12} \Omega^{(2)} P_{12}|\varphi(1,2)\rangle=|\varphi(1,2)\rangle$, and therefore $\Omega^{(2)}|\varphi(2,1)\rangle=|\varphi(2,1)\rangle$.

However, in addition we have to stipulate the conditions for satisfaction of predicate $\Phi_{\Omega^{(2)}}\left(\xi_{1}, \xi_{2}\right)$ by pairs $(1,1)$ and $(2,2)$, and this turns out to be a harder nut to crack. We can suggest the following solution to this problem in the special case when $\Omega^{(2)}$ $=P_{a} \otimes P_{b}$, and $P_{a}$ commutes with $P_{b}$ :

(11) $\Phi_{\Omega^{(2)}}\left(\xi_{1}, \xi_{2}\right)$ is satisfied by pair $(1,1)$ iff the system is in a state $|\varphi(1,2)\rangle$ such that $P_{a} P_{b} \otimes I|\varphi(1,2)\rangle=|\varphi(1,2)\rangle$,

$\Phi_{\Omega^{(2)}}\left(\xi_{1}, \xi_{2}\right)$ is satisfied by pair $(2,2)$ iff the system is in a state $|\varphi(1,2)\rangle$ such that $I \otimes P_{a} P_{b}|\varphi(1,2)\rangle=|\varphi(1,2)\rangle .^{21}$

What justifies the correctness of these stipulation is that in this special case the property represented by projector $P_{a} P_{b}$ is just the ordinary conjunction of one-particle properties represented by $P_{a}$ and $P_{b}$. Thus the satisfaction of predicate $\Phi_{\Omega^{(2)}}\left(\xi_{1}, \xi_{2}\right)$ by pair $(1,1)$ (or $(2,2)$ ) means simply that object 1 (or 2 ) possesses both attributes $P_{a}$ and $P_{b}$, and this is precisely what the above conditions guarantee. However, this characteristic is formally incorrect when $P_{a}$ and $P_{b}$ do not commute, since in that case $P_{a} P_{b}$ is not a projector. Moreover, the proposed method of interpretation cannot be applied to more complex cases, such as for instance when the operator $\Omega^{(2)}$ is equal to

\footnotetext{
20 Following remarks made in the context of (6), we may want to express (8) alternatively in terms of the multi-type variable predicate $\Phi\left(\xi_{1}, \ldots, \xi_{N}, \Omega_{k}, \Phi\right)$. As I consider this alternative notation more cumbersome, I'll nevertheless continue to use the one-type variable predicate $\Phi_{\Omega_{k}}\left(\xi_{1}, \ldots, \xi_{N}\right)$.

21 This is also a solution adopted implicitly in the concrete example used in Muller and Seevinck (2009).
} 
$P_{a} \otimes P_{b}+P_{b} \otimes P_{a}$ (where $P_{a}$ and $P_{b}$ are orthogonal) In that case it simply does not make much sense to ask what the property represented by this symmetric projector would look like when applied 'twice over' to one particle. Speaking figuratively, the 'entanglement' of the property of a two-particle system represented by $P_{a} \otimes P_{b}+P_{b}$ $\otimes P_{a}$ makes it impossible to disentangle it in a way necessary to turn it into a relational property of one particle.

In conclusion, it is possible to define many-argument quantum predicates applicable to sequences of distinct objects; however 'contractions' of such predicates with smaller numbers of arguments are well defined only in special cases.

\section{Absolute discernibility in the quantum theory of many particles}

In this section we will make use of the tools developed in the previous sections in order to formally argue that absolute discernibility is attainable in the quantum-mechanical formalism of many-particle systems, even if these systems can only occupy permutation-invariant states. Let us consider the most general case of $N$ particles, labeled by natural numbers $1, \ldots, N$, and let us suppose that they have been prepared in the product state

$$
|\chi(1, \ldots N)\rangle=\left|a_{1}\right\rangle\left|a_{2}\right\rangle \ldots\left|a_{N}\right\rangle
$$

where each two vectors $\left|a_{i}\right\rangle$ and $\left|a_{j}\right\rangle$ are orthogonal for $i \neq j .{ }^{22}$ Moreover, let $P^{(i)}$ $=\left|a_{i}\right\rangle\left\langle a_{i}\right|$. Now we can define $N$ monadic predicates $\Phi_{i}(\xi)$, where $i=1, \ldots, N$, as follows:

(12) $\Phi_{i}(\xi)$ is satisfied by particle $j$ iff the system is in a state $|\varphi\rangle$ such that $\Omega_{j}^{(i)}|\varphi\rangle=$ $|\varphi\rangle$, where $\Omega_{j}^{(i)}=I \otimes I \ldots \otimes \underbrace{P^{(i)}}_{j} \otimes \ldots \otimes I$.

It is elementary to observe that if the system is in state $|\chi(1, \ldots N)\rangle$ defined above, then for every $i$, the $i$ th particle satisfies predicate $\Phi_{i}(x)$ and only it. Thus, for every $k$, $l, \mathcal{A} \vDash \Phi_{k}(k) \wedge \Phi_{l}(l)$, from which it follows that for every $k \neq l, \mathcal{A} \vDash \Phi_{k}(k) \wedge \neg \Phi_{k}(l)$. Consequently, each formula $\Phi_{i}(x)$ absolutely discerns the $i$ th particle from every other particle. We can express this fact in the following general statement:

$$
\forall \xi_{1} \forall \xi_{2}\left\{\xi_{1} \neq \xi_{2} \rightarrow \bigvee_{k=1}^{N}\left[\Phi_{k}\left(\xi_{1}\right) \wedge \neg \Phi_{k}\left(\xi_{2}\right)\right]\right\}
$$

Applying the method of symmetrization described in Sect. 2, we can reformulate the above 'discernibility' sentence in a permutation-invariant way:

$$
\exists \xi_{1} \ldots \exists \xi_{N}\left\{\rho\left(\xi_{1}, \ldots, \xi_{N}\right) \wedge \bigwedge_{i \neq j}^{N} \bigvee_{k=1}^{N}\left[\Phi_{k}\left(\xi_{i}\right) \wedge \neg \Phi_{k}\left(\xi_{j}\right)\right]\right\}
$$

\footnotetext{
22 Of course I am perfectly aware of the fact that vector $|\chi(1, \ldots, N)\rangle$ is not permutation-invariant, and as such cannot represent states of systems of 'indistinguishable' particles. The reason we are using this example is to formulate a statement expressing the absolute discernibility of objects occupying such a state, in order to translate it later into a fully symmetric language applicable to 'indistinguishable' particles, following Saunders' method described in Sect. 2.
} 
where $\rho\left(\xi_{1}, \ldots, \xi_{N}\right)$, as before, states that there are exactly $N$ distinct objects. Sentence (14) is built with the help of the totally symmetric $N$-argument predicate:

(15) $\operatorname{Abs}\left(\xi_{1}, \ldots, \xi_{N}\right) \equiv \bigwedge_{i \neq j}^{N} \bigvee_{k=1}^{N}\left[\Phi_{k}\left(\xi_{i}\right) \wedge \neg \Phi_{k}\left(\xi_{j}\right)\right]$

Now the question is whether $\operatorname{Abs}\left(\xi_{1}, \ldots, \xi_{N}\right)$ can be constructed independently of definition (15), which is given in terms of non-symmetric predicates $\Phi_{i}(\xi)$. The reason for asking this question is that in languages obeying the condition of permutationinvariance, predicates $\Phi_{i}(\xi)$ are not admissible, so we can hardly use them to define other formulas, even if these formulas themselves turn out to be permutation-invariant. Following the method of interpreting quantum-mechanical predicates laid out in Sect. 3, we will try to find a symmetric projector with the help of which we could formulate truth conditions for predicate $\operatorname{Abs}\left(\xi_{1}, \ldots, \xi_{N}\right)$ independently of (15). We will do this in steps, starting first with formulas $\Phi_{k}\left(\xi_{1}\right) \wedge \neg \Phi_{k}\left(\xi_{2}\right)$. The truth conditions for such compound formulas can be given as follows:

$$
\begin{aligned}
& \Phi_{k}\left(\xi_{1}\right) \wedge \neg \Phi_{k}\left(\xi_{2}\right) \text { is satisfied by pair }(i, j) \text { iff the system is in a state }|\varphi\rangle \text { such } \\
& \text { that } \Omega_{i j}^{(k)}|\varphi\rangle=|\varphi\rangle \text {, where } \Omega_{i j}^{(k)}=I \otimes \ldots \otimes \underbrace{P^{(k)}}_{i} \otimes \ldots \otimes \underbrace{I-P^{(k)}}_{j} \otimes \ldots \otimes I .^{23}
\end{aligned}
$$

Observe that we are interpreting the negation of the monadic predicate $\Phi_{k}(\xi)$ with the help of the projector $I-P^{(k)}$, which is the orthogonal complement of $P^{(k)}$. This is standard practice in so-called quantum logic; however, it stands in conflict with the ordinary, classical interpretation of negation. ${ }^{24}$ Classically, when the negation $\neg \Phi_{k}(\xi)$ is true of $a$, this means that $a$ occupies any state not in the range of $P^{(k)}$. But the quantum-logical interpretation of negation is stronger-it requires that the state occupied by $a$ be orthogonal to the space projected onto by $P^{(k)}$. Quantum logic does not obey the metalogical principle of the excluded middle: the system $a$ may be in a state in which neither $\Phi_{k}(\xi)$ nor its negation is true of $a$. In the current context we may observe that if we followed the classical interpretation of negation, then the formula $\neg \Phi_{k}(\xi)$ would not have an interpretation in terms of any projector, since the set of all vectors in a Hilbert space minus one ray is not a vector space, and cannot form the range of any projector.

Next, we will extend our projector-based interpretation to the disjunctive formula $\bigvee_{k=1}^{N}\left[\Phi_{k}\left(\xi_{1}\right) \wedge \neg \Phi_{k}\left(\xi_{2}\right)\right]:$

(17) $\bigvee_{k=1}^{N}\left[\Phi_{k}\left(\xi_{1}\right) \wedge \neg \Phi_{k}\left(\xi_{2}\right)\right]$ is satisfied by pair $(i, j)$ iff the system is in a state $|\varphi\rangle$ such that $\Gamma_{i j}|\varphi\rangle=|\varphi\rangle$, where $\Gamma_{i j}=\sum_{k=1}^{N} \Omega_{i j}^{(k)}{ }^{25}$

\footnotetext{
23 When $i=j$, it is stipulated, according to the method used in (11), that the corresponding projector has the operator $P^{(k)}\left(I-P^{(k)}\right)=0$ in the $i$ th place of the whole tensor product. From this it follows that the projector is the null operator, and consequently that formula $\Phi_{k}\left(\xi_{1}\right) \wedge \neg \Phi_{k}\left(\xi_{2}\right)$ can never be satisfied by pairs $(i, i)$.

24 For a nice introduction to quantum logic see Hughes (1989, pp. 178-217). The assumption of quantum logic seems necessary here, since otherwise we could not legitimately use the operations of the orthogonal complement and addition of projectors in the appropriate interpretations of formulas of the constructed language. Moreover, the formal result quoted at the end of Sect. 4 can be interpreted as proving the absolute discernibility of quantum particles only under the quantum-logical interpretation of the connectives "not" and "or". This fact raises the question of whether Saunders' theorem can be reinterpreted in terms of quantum logic. I briefly address this issue in Conclusion.

${ }^{25} \Gamma_{i j}$ is a projector thanks to the fact that projectors $\Omega_{i j}^{(k)}$ are mutually orthogonal.
} 
This time we have adopted yet another quantum modification of a classical logical concept, namely that of disjunction. Quantum disjunction is interpreted in terms of the span of vector spaces in the following sense: if sentence $\alpha$ is true if and only if the state vector of the system lies in space $\mathcal{V}_{\alpha}$, and the truth condition for sentence $\beta$ is the same as above with space $\mathcal{V}_{\beta}$ replacing $\mathcal{V}_{\alpha}$, then the quantum disjunction $\alpha \vee \beta$ is true iff the state vector lies in the space that is spanned by $\mathcal{V}_{\alpha}$ and $\mathcal{V}_{\beta}$. This interpretation of disjunction is weaker than classical disjunction based on the notion of set-theoretic sum rather than span. That is, if the state of the system is given as a non-trivial linear combination of states from $\mathcal{V}_{\alpha}$ and $\mathcal{V}_{\beta}$, the quantum disjunction of sentences $\alpha$ and $\beta$ is true but the classical disjunction is false.

Finally, we can present the complete projector corresponding to the symmetric predicate Abs defined in (15):

(18) $\operatorname{Abs}\left(\xi_{1}, \ldots, \xi_{N}\right)$ is satisfied by an $N$-tuple $\left(a_{1}, \ldots, a_{N}\right)$, where $a_{i} \neq a_{j}$ for $i \neq j$, iff the system is in a state $|\varphi\rangle$ such that $\Xi|\varphi\rangle=|\varphi\rangle$, where $\Xi=\prod_{i \neq j}^{N} \Gamma_{i j}=$ $\prod_{i \neq j}^{N} \sum_{k=1}^{N} \Omega_{i j}^{(k)} \cdot 26$

Note that this time the appropriate projector $\Xi$ does not depend on the selected sequence of objects $\left(a_{1}, \ldots, a_{N}\right)$, thanks to the permutation invariance of the corresponding predicate. Also, we may observe that the interpretation of the logical connective of conjunction in terms of the product of (commuting) projectors that underlies definition (18) is equivalent to that adopted in classical logic. The formal reason for this is that the intersection (set-theoretical product) of two vector spaces is itself a vector space.

It can be verified that projector $\Xi$ has the following, simple form (see “Appendix"):

$$
\Xi=\sum_{\sigma} P^{\sigma(1)} \otimes P^{\sigma(2)} \otimes \ldots \otimes P^{\sigma(N)}
$$

Suppose, now, that the system of $N$ 'indistinguishable' particles occupies one of the two following states, depending on whether we are dealing with bosons or fermions:

$$
\begin{aligned}
& \operatorname{Sym}(\mid \chi(1, \ldots, N\rangle)=\sum_{\sigma}\left|a_{\sigma(1)}\right\rangle\left|a_{\sigma(2)}\right\rangle \ldots\left|a_{\sigma(N)}\right\rangle \\
& \operatorname{Anti}(\mid \chi(1, \ldots, N\rangle)=\sum_{\sigma} \operatorname{sgn}(\sigma)\left|a_{\sigma(1)}\right\rangle\left|a_{\sigma(2)}\right\rangle \ldots\left|a_{\sigma(N)}\right\rangle
\end{aligned}
$$

It is straightforward to observe that vectors (20) lie in the range of the operator $\Xi$, as presented in (19). Thus, we can conclude that particles occupying states (20) are absolutely distinguishable by their properties.

\section{Conclusion}

Saunders' theorem goes against the widely-held belief that permutation-invariance and absolute discernibility are irreconcilable. It shows that even in a language that consists

\footnotetext{
26 That operator $\Xi$ is a projector is guaranteed by the fact that projectors $\Gamma_{i j}$ commute. The product of two or more commuting projectors is the projector whose range is the intersection of the range spaces for the factor projectors.
} 
of totally symmetric predicates it is possible to express facts about absolute discernibility of objects. And yet the application of this theorem to the case of quantum particles of the same type is not at all straightforward. The main challenge is to make sure that the totally symmetric predicate which encodes the statement about the absolute discernibility of objects can be expressed within the standard quantum-mechanical formalism that we use to describe systems of many particles. In order to accomplish that, I have proposed a particular way of introducing multi-argument predicates into the language of quantum theory. I have suggested that the satisfaction of a given predicate by a $k$-tuple of objects should be tied to the fact that the state of the system containing these objects lies in the range of a selected projection operator. Given this interpretational rule, I have shown that the symmetric formula encoding the sentence that all objects in the domain are absolutely discernible by one-particle projectors, corresponds to a particular permutation-invariant combination of these projectors. This correspondence ensures that when the system occupies a state represented by a vector within the range of this compound projector, it may be claimed that the individual particles are absolutely discerned by their quantum-mechanical properties.

It has to be stressed that the above result relies on one crucial assumption-namely that the logical connectives used in the symmetric formulation of the condition of absolute discernibility (3) are interpreted quantum-logically rather than classically. It may be argued that this assumption weakens slightly the absolute discernibility claim made at the end of Sect. 4, since this claim cannot be upheld if we decide to use the standard, classical interpretation. Moreover, one can raise the concern that the interpretation of sentence $A b s$ in (1) and its symmetric reformulation (3) following Saunders' method, as expressing the absolute discernibility of objects, implicitly presupposes the classical concepts of disjunction and negation. It is unclear whether we can continue to interpret $A b s$ in the same way when we replace classical connectives with their quantum counterparts. In the end this may indicate that the problem of the absolute discernibility of quantum particles is more intricate than we have presented it in this survey. There are some independent arguments showing that the absolute discernibility of fermions in all states and of bosons in the majority of states is admissible, but these arguments presuppose a substantial change in the adopted interpretation of the quantum-mechanical formalism. In particular, we would have to abandon the doctrine of factorism, i.e. the claim that the factor Hilbert spaces in the symmetric and antisymmetric sections of the $N$-fold tensor product represent states of individual particles. Consequently, the symmetry properties of the states of many particles would no longer be connected with invariance with respect to permutations of particles, but would be treated analogously to gauge symmetries as reflecting the representational redundancy of the mathematical formalism. ${ }^{27}$ Whether we follow this new approach, or continue to use the method based on Saunders' theorem, one thing seems to be cer-

\footnotetext{
27 For more on this approach see Caulton (2014). Caulton presents extensive arguments in favor of his non-factorism based approach to the individuation of quantum particles that we don't have space to discuss here in detail. Suffice it to say that he argues, inter alia, that the standard, factorism-based approach does not produce intuitively correct results when we take the classical limit of the quantum theory of many particles, as well as the quantum field theoretical limit. See also a recent defense of factorism in Leegwater and Muller (2019).
} 
tain: the absolute discernibility of same-type quantum particles by their momentary properties is not a far-fetched concept after all.

Acknowledgements I am grateful to the two anonymous referees for their extensive comments to an earlier version of my paper. The work on this paper was supported by Grant No. 2017/25/B/HS1/00620 from the National Science Centre, Poland.

Open Access This article is licensed under a Creative Commons Attribution 4.0 International License, which permits use, sharing, adaptation, distribution and reproduction in any medium or format, as long as you give appropriate credit to the original author(s) and the source, provide a link to the Creative Commons licence, and indicate if changes were made. The images or other third party material in this article are included in the article's Creative Commons licence, unless indicated otherwise in a credit line to the material. If material is not included in the article's Creative Commons licence and your intended use is not permitted by statutory regulation or exceeds the permitted use, you will need to obtain permission directly from the copyright holder. To view a copy of this licence, visit http://creativecommons.org/licenses/by/4.0/.

\section{Appendix}

In what follows I will give a semi-formal argument aiming to show that any product vector of the form $\left|a_{\sigma(1)}\right\rangle\left|a_{\sigma(2)}\right\rangle \ldots\left|a_{\sigma(N)}\right\rangle$ is an eigenstate of the projector $\Xi$ defined in (18), with corresponding eigenvalue equal 1 , and consequently that the symmetric/antisymmetric combination of such vectors has the same property too. Let us begin with a reformulation of operators $\Omega_{i j}^{(k)}$ composing projector $\Xi$, which we initially defined as follows:

$$
\Omega_{i j}^{(k)}=I \otimes \ldots \otimes \underbrace{P^{(k)}}_{i} \otimes \ldots \otimes \underbrace{I-P^{(k)}}_{j} \otimes \ldots \otimes I .
$$

Given that $I-P^{(k)}=\sum_{l \neq k}^{N} P^{(l)}$, we can rewrite the above projector as the following sum:

$$
\Omega_{i j}^{(k)}=\sum_{l \neq k}^{N} I \otimes \ldots \otimes \underbrace{P^{(k)}}_{i} \otimes \ldots \otimes \underbrace{P^{(l)}}_{j} \otimes \ldots \otimes I,
$$

and, consequently, the projectors $\Gamma_{i j}$ from (17) will have the following form:

$$
\Gamma_{i j}=\sum_{k=1}^{N} \sum_{l \neq k}^{N} I \otimes \ldots \otimes \underbrace{P^{(k)}}_{i} \otimes \ldots \otimes \underbrace{P^{(l)}}_{j} \otimes \ldots \otimes I .
$$

The operator $\Xi$ is calculated by taking the product of all the above expressions for any $i, j$ :

$$
\Xi=\prod_{i, j=1}^{N} \sum_{k=1}^{N} \sum_{l \neq k}^{N} I \otimes \ldots \otimes \underbrace{P^{(k)}}_{i} \otimes \ldots \otimes \underbrace{P^{(l)}}_{j} \otimes \ldots \otimes I
$$


Computing this product directly may seem a daunting task, due to an enormous number of possible combinations, since we are calculating here the product of $N(N-1)$ sums, each of which consists of $N(N-1)$ elements, so the number of different combinations to multiply is the staggering $[N(N-1)]^{N(N-1)}$. However, the result of this gigantic number of multiplications may be easier to predict than it seems. First off, we should notice that the product of two projectors $P^{(i)} P^{(j)}$ gives 0 when $i \neq j$ and $P^{(i)}$ when $i=j$. This actually ensures that each non-zero component in the resulting sum that constitutes projector $\Xi$ will have the form of the tensor product of $N$ projectors $P^{(i)}$ without any identity operators (since for each slot in the tensor product there is some $\Gamma_{i j}$ which has this slot filled by some operator $P^{(k)}$, and no product of these operators can give $I$ ). Moreover, we can easily check that this product has to consist of distinct projectors (no repetitions are allowed). Suppose, to the contrary, that one component in the sum constituting $\Xi$ has the form ... $\otimes \underbrace{P^{(k)}}_{i} \otimes \ldots \otimes \underbrace{P^{(k)}}_{j} \otimes \ldots$. But this would mean that within the components of the sum constituting projector $\Gamma_{i j}$ there is a combination of the form $I \otimes \ldots \otimes \underbrace{P^{(k)}}_{i} \otimes \ldots \otimes \underbrace{P^{(k)}}_{j} \otimes \ldots \otimes I$, and this is impossible, given the way we defined $\Gamma_{i j}$. Hence $\Xi$ must contain only products of projectors $P^{(i)}$ with no repetition.

That such products must occur in the decomposition of $\Xi$ can be shown directly. Let $\alpha_{i j}$ be the following selected component of the sum $\Gamma_{i j}$ :

$$
\alpha_{i j}=I \otimes \ldots \otimes \underbrace{P^{(i)}}_{i} \otimes \ldots \otimes \underbrace{P^{(j)}}_{j} \otimes \ldots \otimes I
$$

It is easy to observe that the product of all $\alpha_{i j}$ 's will be exactly the required combination:

$$
\prod_{i, j=1}^{N} \alpha_{i j}=P^{(1)} \otimes P^{(2)} \otimes \ldots \otimes P^{(N)}
$$

However, projector $\Xi$ is obviously symmetric with respect to permutations of places, hence the sum has to contain all permutations of the above tensor product. Consequently, we have established that $\Xi$ has to have the following, simple form:

$$
\Xi=\sum_{\sigma} P^{\sigma(1)} P^{\sigma(2)} \otimes \ldots \otimes P^{\sigma(N)}
$$

from which it immediately follows that all vectors $\left|a_{\sigma(1)}\right\rangle\left|a_{\sigma(2)}\right\rangle \ldots\left|a_{\sigma(N)}\right\rangle$ lie within the range of $\Xi$. And this in turn proves by linearity that the symmetric and antisymmetric vectors $\sum_{\sigma}\left|a_{\sigma(1)}\right\rangle\left|a_{\sigma(2)}\right\rangle \ldots\left|a_{\sigma(N)}\right\rangle$ and $\sum_{\sigma} \operatorname{sgn}(\sigma)\left|a_{\sigma(1)}\right\rangle\left|a_{\sigma(2)}\right\rangle \ldots\left|a_{\sigma(N)}\right\rangle$ are also eigenvectors of $\Xi$. 


\section{References}

Bigaj, T. (2015a). On discernibility and symmetries. Erkenntnis, 80(1), 117-135.

Bigaj, T. (2015b). Dissecting weak discernibility of quanta. Studies in History and Philosophy of Modern Physics, 50(2015), 43-53.

Butterfield, J. (1993). Interpretation and identity in quantum theory. Studies in History and Philosophy of Science, 24, 443-476.

Caulton, A. (2013). Discerning "indistinguishable" quantum systems. Philosophy of Science, 80, 49-72.

Caulton, A. (2014). Qualitative individuation in permutation-invariant quantum mechanics. arXiv: 1409.0247v1 [quant-ph].

Caulton, A., \& Butterfield, J. (2012). On kinds of indiscernibility in logic and metaphysics. British Journal for the Philosophy of Science, 63, 27-84.

Dieks, D., \& Versteegh, M. (2008). Identical quantum particles and weak discernibility. Foundations of Physics, 38, 923-934.

French, S., \& Redhead, M. (1988). Quantum physics and the identity of indiscernibles. British Journal for the Philosophy of Science, 39, 233-246.

French, S., \& Rickles, D. (2003). Understanding permutation symmetry. In K. Brading \& E. Castellani (Eds.), Symmetries in physics: Philosophical reflections (pp. 212-238). Cambridge: Cambridge University Press.

Huggett, N. (2003). Quarticles and the identity of indiscernibles. In W. K. Brading \& E. Castellani (Eds.), Symmetries in physics (pp. 239-249). Cambridge: Cambridge University Press.

Huggett, N., \& Norton, J. (2014). Weak discernibility for quanta, the right way. British Journal for the Philosophy of Science, 65, 39-58.

Hughes, R. I. G. (1989). The structure and interpretation of quantum mechanics. Cambridge, MA: Harvard University Press.

Ketland, J. (2006). Structuralism and the identity of indiscernibles. Analysis, 66, 303-315.

Ketland, J. (2011). Identity and indiscernibility. The Review of Symbolic Logic, 4(2), 171-185.

Ladyman, J., Linnebo, O., \& Pettigrew, R. (2012). Identity and discernibility in philosophy and logic. The Review of Symbolic Logic, 5, 162-186.

Leegwater, G., \& Muller, F. A. (2019). "The case against factorism”, forthcoming in Journal for General Philosophy of Science.

Muller, F. A. (2011). Withering away, weakly. Synthese, 180, 223-233.

Muller, F. A. (2015). The rise of relationals. Mind, 124, 201-237.

Muller, F. A., \& Saunders, S. (2008). Discerning fermions. British Journal for the Philosophy of Science, 59, 499-548.

Muller, F. A., \& Seevinck, M. P. (2009). Discerning elementary particles. Philosophy of Science, 76, 179-200.

Redhead, M., \& Teller, P. (1992). Quantum physics and the identity of the indiscernibles. British Journal for the Philosophy of Science, 43, 201-218.

Saunders, S. (2003). Physics and Leibniz's principles. In K. Brading \& E. Castellani (Eds.), Symmetries in physics: Philosophical reflections (pp. 289-307). Cambridge: Cambridge University Press.

Saunders, S. (2006a). Are quantum particles objects? Analysis, 66, 52-63.

Saunders, S. (2006b). On the explanation of quantum statistics. Studies in the History and Philosophy of Modern Physics, 37, 192-211.

Saunders, S. (2013). Indistinguishability. In R. Batterman (Ed.), Oxford handbook of philosophy of physics (pp. 340-380). Oxford: Oxford University Press.

Publisher's Note Springer Nature remains neutral with regard to jurisdictional claims in published maps and institutional affiliations. 